\title{
REALIMENTAÇÃO DA DERIVADA DOS ESTADOS EM SISTEMAS MULTIVARIÁVEIS LINEARES USANDO LMIS
}

\author{
Flávio Andrade Faria* \\ flaviof15eyahoo.com.br
}

\author{
Edvaldo Assunção* \\ edvaldo@dee.feis.unesp.br
}

\author{
Marcelo C. M. Teixeira* \\ marcelo@dee.feis.unesp.br
}

*UNESP - Universidade Estadual Paulista

Departamento de Engenharia Elétrica, Campus de Ilha Solteira

Avenida Brasil, 56, 15385-000, Ilha Solteira, SP, Brasil

\begin{abstract}
In some practical problems, for instance in the control systems for the suppression of vibration in mechanical systems, the state-derivative signals are easier to obtain than the state signals. A new technique for stabilization of linear multivariable systems by state-derivative feedback is proposed. The proposed method is described in Linear Matrix Inequalities (LMIs) and assumes that the plant is a controllable system with poles different from zero. It can include design constraints such as: decay rate, bounds on output peak and structural failures. An optimization procedure to obtain the controller gain with constraint in the norm is also proposed. The efficiency of design procedure is illustrated through three numerical examples.
\end{abstract}

KEYWORDS: Linear Matrix Inequalities (LMIs), StateDerivative Feedback, Decay Rate, Bounds on Output Peak, Structural Failures.

\section{RESUMO}

Em alguns problemas práticos, por exemplo em sistemas mecânicos para controle de vibrações, é mais fácil obter o sinal da derivada dos estados do que os sinais dos estados. Uma nova técnica para estabilização de sistemas

Artigo submetido em 05/10/2006 (Id:750)

Revisado em 13/02/2007, 17/09/2007, 10/04/2008 e em 27/11/2008

Aceito sob recomendação do Editor Associado Prof. Liu Hsu multivariáveis lineares sob realimentação da derivada dos estados é proposta. Este método é baseado em LMIs e supõe que a planta é controlável e não possui pólos na origem. Ele permite a inserção de restrições de projeto tais como: taxa de decaimento, restrições na saída do sistema e a existência de falhas estruturais. É proposta também uma metodologia de otimização que obtém os ganhos dos controladores com menores normas. A eficiência do procedimento de projeto é ilustrada através da solução de três exemplos de aplicação.

PALAVRAS-CHAVE: Desigualdades Matriciais Lineares (LMIs), Realimentação da Derivada dos Estados, Taxa de Decaimento, Restrições na Saída do Sistema, Falhas Estruturais.

\section{INTRODUÇÃO}

A realimentação de sistemas constitui a base da teoria de controle e uma técnica comumente usada é a realimentação dos estados (Ogata, 2000; Dorf and Bishop, 2001; Chen, 1999). Neste trabalho propõe-se novos resultados usando uma outra técnica de realimentação, que é a realimentação da derivada dos estados.

Em Lewis and Syrmos (1991) é feita uma abordagem mostrando que algumas vezes esse tipo de realimentação é essencial para que o sistema controlado apresente um desempenho satisfatório. Contudo a motivação para esse trabalho, reside no fato que existem problemas práticos em 
que a derivada dos estados é mais fácil de se obter do que os sinais dos estados. Como exemplo pode-se citar sistemas nos quais os sensores usados são acelerômetros. A partir da aceleração é possível obter a velocidade com boa precisão, porém se o sinal for integrado mais uma vez o resultado terá um acúmulo de erros e não representará o deslocamento satisfatoriamente (Abdelaziz and Valášek, 2004), daí os sinais usados para realimentar esses sistemas são: a aceleração e a velocidade e estes são justamente as derivadas da velocidade e da posição, que representam as variáveis de estado do sistema. Devido ao seu baixo custo os acelerômetros têm sido cada vez mais usados pela indústria. As aplicações mais comuns são em controle de oscilações de sistemas mecânicos (Abdelaziz and Valášek, 2004; S. K. Kwak and G. Washington and R. K. Yedavalli, 2002a), na indústria automobilística (E. Reithmeier and G. Leitmann, 2003), na engenharia aeronáutica (S. K. Kwak and G. Washington and R. K. Yedavalli, 2002b) e na engenharia civil (Y. F. Duan and Y. Q. Ni and J. M. Ko, 2005). Técnicas de projeto de controle, para sistemas lineares usando realimentação da derivada de estados, têm sido exploradas sob várias metodologias. Em (Abdelaziz and Valášek, 2004) é apresentada uma solução para plantas com uma entrada e uma saída (SISO). Os autores desenvolveram uma fórmula de Ackermann generalizada. Usando o conceito de espaço de estados recíproco (do inglês, Reciprocal State Space $(R S S)$ ), um projeto para controle ótimo de sistemas lineares foi proposto por (S. K. Kwak and G. Washington and R. K. Yedavalli, 2002a). Em (Teixeira, Assunção, Cardim and Covacic, 2006; Cardim et al., 2008) os autores apresentaram uma nova forma de se obter o controlador da realimentação da derivada dos estados a partir da realimentação de estados usual.

Alguns autores (Bunse-Gerstner et al., 1992; A. BunseGerstner and Nichols, 1999; Duan et al., 1999; GarciaPlanas, 2003) têm usado a realimentação derivativa e proporcional $(\mathbf{u}(t)=\mathbf{L} \mathbf{x}(t)-\mathbf{K} \dot{\mathbf{x}}(t))$ no estudo de sistemas descritores,

$$
\mathbf{E} \dot{\mathbf{x}}(t)=\mathbf{A x}(t)+\mathbf{B u}(t)
$$

Ao contrário das técnicas presentes em (Abdelaziz and Valášek, 2004; Teixeira, Assunção, Cardim and Covacic, 2006), a metodologia proposta nesse trabalho não necessita de técnicas de controle auxiliares para a solução do problema. O objetivo desse trabalho é encontrar condições para a estabilidade de sistemas lineares usando apenas a realimentação da derivada dos estados, os resultados propostos são aplicáveis em sistemas lineares com múltiplas entradas e múltiplas saídas (em inglês, Multi-Input MultiOutput MIMO) e são obtidos na forma da solução de Desigualdades Matriciais Lineares (LMIs, do inglês Linear Matrix Inequalities). LMIs quando factíveis, são facilmente resolvidas em microcomputadores usando softwares de programação matemática, como o MATLAB (Gahinet et al., 1995) ou o LMISol (de Oliveira et al., 1997), e ainda permitem que os resultados encontrados, em geral, sejam facilmente estendidos para sistemas que possuem incertezas nos parâmetros da planta ou que estejam sujeitos à falhas estruturais (Isermann and Ballé, 1997; Isermann, 1997). Devido a essas flexibilidades, LMIs têm sido usadas para resolver diversos tipos de problemas de controle (Teixeira et al., 2003; Teixeira et al., 2005; Teixeira, Covacic and Assunção, 2006; Assunção, Marchesi, Teixeira and Peres, 2007; Assunção, Andrea and Teixeira, 2007; Faria et al., 2009).

Neste trabalho são propostas condições necessárias e suficientes, baseadas em LMI, para que sistemas multivariáveis lineares realimentados com a derivada dos estados sejam assintoticamente estáveis. Se a planta possui restrições de desempenho, então nem sempre a estabilização é suficiente. Para resolver este caso, foram adicionadas as restrições de taxa de decaimento e restrição de saída no projeto do controlador. Também são apresentadas condições suficientes para o projeto de sistemas com incertezas politópicas ou sujeitos a falhas estruturais.

Restrições de desempenho atuam no transitório do sistema, mas a solução encontrada nem sempre é implementável. Isso pode acontecer nos problemas em que a magnitude dos parâmetros do controlador $\mathbf{K}$ deve ser limitada para viabilizar sua implementação prática. Esse problema é resolvido adicionando um critério de minimização no projeto do controlador. O critério proposto é simples e usa o conceito de norma matricial (Meyer, 2000; Boyd et al., 1994), para a obtenção de uma solução $\mathbf{K}$ apropriada.

A fim de mostrar a viabilidade e eficiência da técnica proposta, alguns exemplos numéricos são apresentados.

\section{REALIMENTAÇÃO DA DERIVADA DOS ESTADOS EM SISTEMAS LINEARES}

Considere um sistema controlável multivariável linear e invariante no tempo, representado na forma de espaço de estados

$$
\begin{aligned}
\dot{\mathbf{x}}(t) & =\mathbf{A} \mathbf{x}(t)+\mathbf{B} \mathbf{u}(t), \\
\mathbf{y}(t) & =\mathbf{C} \mathbf{x}(t),
\end{aligned}
$$

sendo $\mathbf{x}(t) \in \mathbb{R}^{n}, \mathbf{A} \in \mathbb{R}^{n \times n}, \mathbf{B} \in \mathbb{R}^{n \times m}, \mathbf{u}(t) \in \mathbb{R}^{m}$, $\mathbf{C} \in \mathbb{R}^{r \times n}$ e $\mathbf{y}(t) \in \mathbb{R}^{r}$.

Supondo que o sistema (1) não apresenta pólos na origem $(\operatorname{det}(\mathbf{A}) \neq 0)$. O objetivo é encontrar uma matriz constante $\mathbf{K} \in \mathbb{R}^{m \times n}$, tal que, ao realimentar o sistema (1) com a entrada de controle

$$
\mathbf{u}(t)=-\mathbf{K} \dot{\mathbf{x}}(t)
$$


o sistema em malha fechada, dado por (1) e (2), seja assintoticamente estável e a matriz $(\mathbf{I}+\mathbf{B K})$ seja invertível $(\operatorname{det}(\mathbf{I}+\mathbf{B K}) \neq 0)$. Daí o sistema em malha fechada pode ser representado da seguinte forma:

$$
\begin{aligned}
\dot{\mathbf{x}}(t)=\mathbf{A} \mathbf{x}(t)-\mathbf{B} \mathbf{K} \dot{\mathbf{x}}(t) & \Leftrightarrow(\mathbf{I}+\mathbf{B K}) \dot{\mathbf{x}}(t)=\mathbf{A} \mathbf{x}(t) \\
& \Leftrightarrow \dot{\mathbf{x}}(t)=(\mathbf{I}+\mathbf{B K})^{-\mathbf{1}} \mathbf{A} \mathbf{x}(t) .
\end{aligned}
$$

Em (Teixeira, Assunção, Cardim and Covacic, 2006; Cardim et al., 2008) os autores mostram que se o sistema (1) não tem pólos na origem, então pode-se obter o ganho $\mathbf{K}$ da realimentação da derivada dos estados (2), a partir da realimentação de estados usual $(\mathbf{u}(t)=-\mathbf{L x}(t))$. Uma das grandes vantagens dessa metodologia é que ela permite o uso de técnicas já consagradas na literatura para a obtenção do ganho K. A técnica consiste em substituir $\mathbf{u}(t)=-\mathbf{L x}(t)$ em (1), obter o sistema em malha fechada

$$
\dot{\mathbf{x}}(t)=(\mathbf{A}-\mathbf{B L}) \mathbf{x}(t) .
$$

Resolver esse problema através de uma das inúmeras técnicas já conhecidas na literatura, tais como: alocação de pólos, LQR, ou LMIs. E então obter o ganho $\mathbf{K}$ da realimentação da derivada dos estados através da igualdade:

$$
\mathbf{K}=\mathbf{L}(\mathbf{A}-\mathbf{B L})^{-1} .
$$

Uma outra metodologia pode ser encontrada em (Abdelaziz and Valášek, 2004), onde os autores apresentam uma técnica para alocação de pólos com realimentação da derivada dos estados, usando uma generalização da fórmula de Ackermann. Assim como a fórmula de Ackermann clássica, os resultados apresentados necessitam que sejam encontrados os pólos de interesse através de uma outra técnica de controle para a obtenção do controlador $\mathbf{K}$. Essa metodologia não pode ser aplicada em sistemas multivariáveis $(m>1)$.

Embora eficientes, as duas metodologias citadas anteriormente não resolvem sistemas incertos (Veja Seção 3.3). Estudos recentes, mostram que a alocação de pólos de sistemas lineares incertos usando realimentação da derivada dos estados pode ser realizada através de LMIs (Faria et al., 2009). A metodologia apresentada usa o conceito de $\mathcal{D}$-estabilidade para a alocação dos pólos do vértices do sistema incerto em uma determinada região do plano complexo.

Nas próximas seções são apresentados resultados que podem ser usados na solução de sistemas incertos e multivariáveis $(m \geq 1)$, para a obtenção do controlador $\mathbf{K}$ que estabiliza o sistema (3).

\section{PROJETO DO CONTROLADOR}

Nesta seção são propostas condições necessárias e suficientes para que o sistema (3) seja assintoticamente estável. Adicionalmente, incorpora-se as restrições de taxa de decaimento e restrição de saída no projeto do controlador a fim de melhorar o desempenho do sistema.

\subsection{Condição de estabilidade}

O projeto do controlador é realizado usando o conceito de estabilidade assintótica, através da análise da existência de uma função de Lyapunov (Ogata, 2000; Dorf and Bishop, 2001; Chen, 1999; Boyd et al., 1994) para o sistema (3). O próximo teorema apresenta condições necessárias e suficientes para que (3) seja assintoticamente estável. Durante a prova do teorema usa-se a seguinte propriedade de soma de matrizes:

Propriedade 1 Para toda matriz $\mathbf{M}$ não simétrica $\left(\mathbf{M} \neq \mathbf{M}^{\prime}\right)$, se $\mathbf{M}+\mathbf{M}^{\prime}<0$, então $\mathbf{M}$ é invertível.

Teorema 1 Existe uma matriz $\mathbf{K}$ tal que $(\mathbf{I}+\mathbf{B K}) e ́$ invertível e o sistema (3) é assintoticamente estável se e somente se, existe uma matriz simétrica $\mathbf{Q} \in \mathbb{R}^{n \times n}$ e uma matriz $\mathbf{Y} \in \mathbb{R}^{m \times n}$, satisfazendo o seguinte conjunto de LMIs:

$$
\begin{gathered}
\mathbf{A Q}+\mathbf{Q A}^{\prime}+\mathbf{A} \mathbf{Y}^{\prime} \mathbf{B}^{\prime}+\mathbf{B Y A}^{\prime}<0 \\
\mathbf{Q}>0 .
\end{gathered}
$$

Se as inequações (5) e (6) são factíveis, então um controlador que resolve o problema pode ser dado por:

$$
\mathbf{K}=\mathbf{Y Q}^{-\mathbf{1}} \text {. }
$$

Prova: (Suficiência) Suponha que existem Q e $\mathbf{Y}$ satisfazendo (5) e (6).

Fazendo as substituições de variáveis $\mathbf{Q}=\mathbf{P}^{-\mathbf{1}}$, $\mathbf{Y}=\mathbf{K} \mathbf{P}^{-1}$ e substituindo em (5) obtém-se

$$
\mathbf{A} \mathbf{P}^{-\mathbf{1}}(\mathbf{I}+\mathbf{B K})^{\prime}+(\mathbf{I}+\mathbf{B K}) \mathbf{P}^{-\mathbf{1}} \mathbf{A}^{\prime}<0 .
$$

Usando a Propriedade 1 em (8) tem-se que a matriz $(\mathbf{I}+\mathbf{B K}) \mathbf{P}^{-\mathbf{1}} \mathbf{A}^{\prime}$ é invertível. Portanto as matrizes $(\mathbf{I}+\mathbf{B K})$ e $\mathbf{A}^{\prime}$ também são invertíveis, daí multiplique (8) à esquerda por $\mathbf{P}(\mathbf{I}+\mathbf{B K})^{-\mathbf{1}}$ e à direita por $\left[(\mathbf{I}+\mathbf{B K})^{\prime}\right]^{-\mathbf{1}} \mathbf{P}$ e obtenha

$$
\mathbf{P}(\mathbf{I}+\mathbf{B K})^{-\mathbf{1}} \mathbf{A}+\mathbf{A}^{\prime}\left[(\mathbf{I}+\mathbf{B K})^{\prime}\right]^{-\mathbf{1}} \mathbf{P}<0 .
$$

Note que a inequação (9) é equivalente a função de Lyapunov para o sistema (3). Por hipótese tem-se que $\mathbf{P}=\mathbf{Q}^{\mathbf{- 1}}>0$. Daí segue que, se as LMIs (5) e (6) são factíveis, então existe 
uma matriz $\mathbf{P}$ satisfazendo as condições de Lyapunov para o sistema (3). Logo (3) é assintoticamente estável.

(Necessidade) Para provar a necessidade note que se (3) é assintóticamente estável, então existe $\mathbf{P}>0$ satisfazendo as condições de Lyapunov. Logo as inequações (5) e (6) são factíveis.

Pelo Teorema 1 conclui-se que o estudo da estabilidade assintótica do sistema (3) é equivalente ao estudo da factibilidade das LMIs (5) e (6). Isso facilita o estudo de estabilidade do sistema, pois LMIs quando factíveis são facilmente resolvidas através de softwares de programação matemática. Dentre os quais citamos o MATLAB (Gahinet et al., 1995) que é o mais usado e o LMISol (de Oliveira et al., 1997) que é de domínio público.

A estabilidade do sistema (3) nem sempre é suficiente, pois existem projetos que possuem restrições de desempenho. A modelagem em LMIs permite que algumas dessas restrições sejam adicionadas de maneira simples ao projeto do controlador. Por exemplo à restrição de taxa de decaimento.

\subsection{Estabilidade com restrição de taxa de decaimento}

Em (Boyd et al., 1994) a taxa de decaimento é definida como sendo o maior número real $\gamma, \gamma>0$, tal que,

$$
\lim _{t \rightarrow \infty} e^{\gamma t}|| \mathbf{x}(t) \|=0,
$$

para toda solução $\mathbf{x}(t)$ de (3), $t \geq 0$.

A restrição de taxa de decaimento é usada para diminuir o transitório do sistema. O próximo teorema encontra condições necessárias e suficientes para que (3) tenha taxa de decaimento maior que $\gamma$.

Teorema 2 Existe uma matriz $\mathbf{K}$ tal que $(\mathbf{I}+\mathbf{B K}) e ́$ invertível e o sistema (3) é assintoticamente estável com taxa de decaimento maior que $\gamma$ se e somente se, existe uma matriz simétrica $\mathbf{Q} \in \mathbb{R}^{n \times n}$ e uma matriz $\mathbf{Y} \in \mathbb{R}^{m \times n}$ satisfazendo o seguinte conjunto de LMIs:

$$
\left[\begin{array}{cc}
\mathbf{A Q}+\mathbf{Q A}^{\prime}+\mathbf{B Y A}^{\prime}+\mathbf{A} \mathbf{Y}^{\prime} \mathbf{B}^{\prime} & \mathbf{Q}+\mathbf{B Y} \\
\mathbf{Q}+\mathbf{Y}^{\prime} \mathbf{B}^{\prime} & -\frac{\mathbf{Q}}{2 \gamma}
\end{array}\right]<0,
$$

$$
\mathbf{Q}>0 \text {. }
$$

Se as inequações (10) e (11) são factíveis, então um controlador que resolve o problema pode ser dado por:

$$
\mathbf{K}=\mathbf{Y Q}^{-\mathbf{1}} \text {. }
$$

Prova: (Necessidade) Inserir a restrição de taxa de decaimento no sistema (3) é equivalente a trocar a inequação $\dot{V}(\mathbf{x}(t))<0$ por (Boyd et al., 1994):

$$
\dot{V}(\mathbf{x}(t))<-2 \gamma V(\mathbf{x}(t)),
$$

sendo $V(\mathbf{x}(t))=\mathbf{x}^{\prime}(t) \mathbf{P x}(t)$ uma função quadrática de Lyapunov. Substituindo (3) em $\dot{V}(\mathbf{x}(t))$ tem-se

$$
\begin{aligned}
\dot{V}(\mathbf{x}(t)) & =\mathbf{x}^{\prime}(t) \mathbf{P} \dot{\mathbf{x}}(t)+\dot{\mathbf{x}}^{\prime}(t) \mathbf{P} \mathbf{x}(t) \\
& =\mathbf{x}^{\prime}(t) \mathbf{P}(\mathbf{I}+\mathbf{B K})^{-\mathbf{1}} \mathbf{A} \mathbf{x}(t)+ \\
& \mathbf{x}^{\prime}(t) \mathbf{A}^{\prime}\left[(\mathbf{I}+\mathbf{B K})^{-\mathbf{1}}\right]^{\prime} \mathbf{P} \mathbf{x}(t) .
\end{aligned}
$$

Então de (13) segue que

$$
\begin{aligned}
& \mathbf{x}^{\prime}(t) \mathbf{P}(\mathbf{I}+\mathbf{B K})^{-\mathbf{1}} \mathbf{A} \mathbf{x}(t)+ \\
& \quad \mathbf{x}^{\prime}(t) \mathbf{A}^{\prime}\left[(\mathbf{I}+\mathbf{B K})^{-\mathbf{1}}\right]^{\prime} \mathbf{P} \mathbf{x}(t)<-2 \gamma \mathbf{x}^{\prime}(t) \mathbf{P} \mathbf{x}(t) \\
& \Leftrightarrow \mathbf{P}(\mathbf{I}+\mathbf{B K})^{-\mathbf{1}} \mathbf{A}+\mathbf{A}^{\prime}\left[(\mathbf{I}+\mathbf{B K})^{-\mathbf{1}}\right]^{\prime} \mathbf{P}<-2 \gamma \mathbf{P} .
\end{aligned}
$$

Decorre diretamente de (14) que $\mathbf{P}(\mathbf{I}+\mathbf{B K})^{-\mathbf{1}} \mathbf{A}+$ $\mathbf{A}^{\prime}\left[(\mathbf{I}+\mathbf{B K})^{-\mathbf{1}}\right]^{\prime} \mathbf{P}<0$, então usando a Propriedade 1 conclui-se que a matriz $\mathbf{P}(\mathbf{I}+\mathbf{B K})^{-1}$ é invertível. Daí multiplique (14) à esquerda por $(\mathbf{I}+\mathbf{B K}) \mathbf{P}^{-1}$ e à direita por $\mathbf{P}^{-\mathbf{1}}(\mathbf{I}+\mathbf{B K})^{\prime}$ e obtenha

$$
\begin{gathered}
\mathbf{A} \mathbf{P}^{-\mathbf{1}}(\mathbf{I}+\mathbf{B K})^{\prime}+(\mathbf{I}+\mathbf{B K}) \mathbf{P}^{-\mathbf{1}} \mathbf{A}^{\prime}- \\
(\mathbf{I}+\mathbf{B K}) \mathbf{P}^{-\mathbf{1}}(-2 \gamma \mathbf{P}) \mathbf{P}^{-\mathbf{1}}\left[(\mathbf{I}+\mathbf{B K})^{\prime}\right]<0 \\
\Leftrightarrow \mathbf{A}\left(\mathbf{P}^{-\mathbf{1}}+\mathbf{B K} \mathbf{P}^{-\mathbf{1}}\right)^{\prime}+\left(\mathbf{P}^{-\mathbf{1}}+\mathbf{B K} \mathbf{P}^{-\mathbf{1}}\right) \mathbf{A}^{\prime}- \\
\left(\mathbf{P}^{-\mathbf{1}}+\mathbf{B K P}^{-\mathbf{1}}\right)(-2 \gamma \mathbf{P})\left[\left(\mathbf{P}^{-\mathbf{1}}+\mathbf{B K} \mathbf{P}^{-\mathbf{1}}\right)^{\prime}\right]<0 .
\end{gathered}
$$

Aplique o complemento de Schur (Boyd et al., 1994; Assunção and Teixeira, 2001) na inequação acima e obtenha a inequação (16). Definindo $\mathbf{Q}=\mathbf{P}^{-\mathbf{1}}$ e $\mathbf{Y}=\mathbf{K} \mathbf{P}^{-\mathbf{1}}$, conclui-se que as inequações (16) e (10) são equivalentes. Como $\mathbf{P}$ é matriz da função de Lyapunov, segue que $\mathbf{P}>$ $0 \Leftrightarrow \mathbf{P}^{-1}=\mathbf{Q}>0$. Portanto quando (10) e (11) são factíveis, o sistema (3) é assintoticamente estável com taxa de decaimento superior a $\gamma$. E o controlador $\mathbf{K}$ pode ser dado por (12).

(Suficiência) Como mencionado anteriormente, se (10) e (11) são factíveis, então existe $\mathbf{P}>0$ satisfazendo a condição $\dot{V}(\mathbf{x}(t))<-2 \gamma V(\mathbf{x}(t))$, daí (3) é assintoticamente estável com taxa de decaimento superior a $\gamma$.

Até o momento, foram apresentadas condições necessárias e suficientes para o projeto do controlador de sistemas lineares determinísticos. Porém já é conhecido na literatura (Boyd et al., 1994) que projetos de controle usando LMIs podem, de um modo geral, ser facilmente adaptados para sistemas incertos. 


$$
\left[\begin{array}{cc}
\mathbf{A}\left(\mathbf{P}^{-\mathbf{1}}+\mathbf{B K \mathbf { P } ^ { - 1 }}\right)^{\prime}+\left(\mathbf{P}^{-\mathbf{1}}+\mathbf{B K} \mathbf{P}^{-\mathbf{1}}\right) \mathbf{A}^{\prime} & \mathbf{P}^{-\mathbf{1}}+\mathbf{B K} \mathbf{P}^{-\mathbf{1}} \\
\left(\mathbf{P}^{-\mathbf{1}}+\mathbf{B K} \mathbf{P}^{-1}\right)^{\prime} & -\frac{\mathbf{P}^{-1}}{2 \gamma}
\end{array}\right]<0
$$

\subsection{Condição de estabilidade sistemas lineares incertos}

Considere um sistema controlável linear e invariante no tempo que possui incertezas politópicas, descrito por:

$$
\begin{gathered}
\dot{x}(t)=\sum_{i=1}^{p} \alpha_{i} \mathbf{A}_{\mathbf{i}} \mathbf{x}(t)+\mathbf{B u}(t), \\
\mathrm{e} \\
\alpha_{i} \geq 0, \quad i=1, \cdots, p, \quad \sum_{i=1}^{p} \alpha_{i}=1,
\end{gathered}
$$

sendo $p$ a quantidade de vértices do politopo em $\mathbf{A}$ e $\alpha_{i}$ números reais desconhecidos.

Aplicando a técnica proposta por (Teixeira, Assunção, Cardim and Covacic, 2006) no sistema (17), obtém-se a igualdade:

$$
\mathbf{K}=\mathbf{L}\left(\sum_{i=1}^{p} \alpha_{i} \mathbf{A}_{\mathbf{i}}-\mathbf{B L}\right)^{-1}
$$

Observe na equação (19) que o controlador obtido, depende dos números $\alpha_{i}$. Na prática esses valores são desconhecidos. Dessa forma, a técnica proposta por (Teixeira, Assunção, Cardim and Covacic, 2006) não pode ser usada para a solução do sistema (17). Por motivos semelhantes, a fórmula de Ackermann generalizada proposta por (Abdelaziz and Valášek, 2004) também não pode ser aplicada em (17).

O projeto de controladores com LMIs pode resolver o sistema (17), considerando apenas os valores dos vértices do politopo da matriz. O próximo teorema propõe condições suficientes para a estabilidade de (3), substituindo o sistema determinístico (1) pelo sistema incerto (17).

Teorema 3 Existe uma matriz $\mathbf{K}$ tal que $(\mathbf{I}+\mathbf{B K})$ é invertível e o sistema (17) é assintoticamente estável se existe uma matriz simétrica $\mathbf{Q} \in \mathbb{R}^{n \times n}$ e uma matriz $\mathbf{Y} \in \mathbb{R}^{m \times n}$ satisfazendo o seguinte conjunto de LMIs:

$$
\begin{gathered}
\mathbf{A}_{\mathbf{i}} \mathbf{Q}+\mathbf{Q A}_{\mathbf{i}}^{\prime}+\mathbf{A}_{\mathbf{i}} \mathbf{Y}^{\prime} \mathbf{B}^{\prime}+\mathbf{B} \mathbf{Y} \mathbf{A}_{\mathbf{i}}^{\prime}<\mathbf{0}, \quad i=1,2, \ldots, p, \\
\mathbf{Q}>\mathbf{0} .
\end{gathered}
$$

Se as inequações (20) e (21) são factíveis, então um controlador que resolve o problema pode ser dado por:

$$
\mathbf{K}=\mathbf{Y Q}^{-\mathbf{1}} \text {. }
$$

Prova: Supondo que (20) e (21) são factíveis. Segue de (18) e (20) que

$$
\begin{aligned}
& \sum_{i=1}^{p} \alpha_{i}\left[\mathbf{A}_{\mathbf{i}} \mathbf{Q}+\mathbf{Q A}_{\mathbf{i}}^{\prime}+\mathbf{A}_{\mathbf{i}} \mathbf{Y}^{\prime} \mathbf{B}+\mathbf{B} \mathbf{Y} \mathbf{A}_{\mathbf{i}}^{\prime}\right] \\
= & \left(\sum_{i=1}^{p} \alpha_{i} \mathbf{A}_{\mathbf{i}}\right) \mathbf{Q}+\mathbf{Q}\left(\sum_{i=1}^{p} \alpha_{i} \mathbf{A}_{\mathbf{i}}{ }^{\prime}\right) \\
& +\left(\sum_{i=1}^{p} \alpha_{i} \mathbf{A}_{\mathbf{i}}\right) \mathbf{Y}^{\prime} \mathbf{B}^{\prime}+\mathbf{B Y}\left(\sum_{i=1}^{p} \alpha_{i} \mathbf{A}_{\mathbf{i}}{ }^{\prime}\right)<\mathbf{0 .}
\end{aligned}
$$

Substituindo $\mathbf{A}=\alpha_{1} \mathbf{A}_{1}+\ldots+\alpha_{p} \mathbf{A}_{\mathbf{p}}$ em (5), tem-se que quando (20) e (21) são factíveis, o sistema incerto (17), satisfaz as LMIs do Teorema 1. Logo (17) é assintoticamente estável.

Da maneira similar pode-se estender o resultado do Teorema 2 para sistemas incertos.

Teorema 4 Existe uma matriz $\mathbf{K}$ tal que $(\mathbf{I}+\mathbf{B K}) e ́$ invertivel e o sistema (17) é assintoticamente estável com taxa de decaimento maior que $\gamma$ se existe uma matriz simétrica $\mathbf{Q} \in \mathbb{R}^{n \times n}$ e uma matriz $\mathbf{Y} \in \mathbb{R}^{m \times n}$ satisfazendo o seguinte conjunto de LMIs:

$$
\left[\begin{array}{cc}
\mathbf{A}_{\mathbf{i}} \mathbf{Q}+\mathbf{Q} \mathbf{A}_{\mathbf{i}}^{\prime}+\mathbf{B Y A}_{\mathbf{i}}^{\prime}+\mathbf{A}_{\mathbf{i}} \mathbf{Y}^{\prime} \mathbf{B}^{\prime} & \mathbf{Q}+\mathbf{B Y} \\
\mathbf{Q}+\mathbf{Y}^{\prime} \mathbf{B}^{\prime} & -\frac{\mathbf{Q}}{2 \gamma}
\end{array}\right]<0,
$$

$$
\mathbf{Q}>0 \text {. }
$$

Se as inequações (23) e (24) são factíveis, então um controlador que resolve o problema pode ser dado por:

$$
\mathbf{K}=\mathbf{Y Q}^{-\mathbf{1}} \text {. }
$$

Prova: A prova segue diretamente das provas dos Teoremas 2 e 3.

Certos tipos de falhas estruturais podem ser representadas como um sistema (17) e então serem resolvidas usando os Teoremas 3 ou 4. Na Seção 4, um exemplo prático é resolvido sob essa metodologia.

O projeto de controle através de LMIs além permitir o tratamento tanto de sistemas determinísticos quanto de sistemas incertos, também permite a inclusão de diversas restrições de desempenho, por exemplo restrição de saída no sistema (Boyd et al., 1994). 


\subsection{Condição de estabilidade com saída limitada}

Quando o sistema (3) é assintoticamente estável, o seu valor da saída $\mathbf{y}(t)=\mathbf{C x}(t)$ pode ser limitado da seguinte forma:

$$
\max \|\mathbf{y}(t)\|=\max \sqrt{\mathbf{y}^{\prime}(t) \mathbf{y}(t)}<\xi_{0},
$$

sendo $t \geq 0$ e $\xi_{0}$ uma constante real positiva préestabelecida. De (Boyd et al., 1994) a equação (26) é satisfeita quando as seguintes LMIs são factíveis:

$$
\begin{aligned}
& {\left[\begin{array}{cc}
1 & \mathbf{x}(0)^{\prime} \\
\mathbf{x}(0) & \mathbf{Q}
\end{array}\right]>0,} \\
& {\left[\begin{array}{cc}
\mathbf{Q} & \mathbf{Q} \mathbf{C}^{\prime} \\
\mathbf{C Q} & \xi_{0}^{2} \mathbf{I}
\end{array}\right]>0 .}
\end{aligned}
$$

sendo $\mathbf{y}(t) \in \mathbb{R}^{r}, \mathbf{C} \in \mathbb{R}^{r \times n}$ e $\mathbf{x}(0)$ a condição inicial do sistema.

Então o estudo de estabilidade assintótica do sistema (3) com saída limitada, pode ser realizado adicionando as LMIs (27) e (28) ao Teorema 1 ou ao Teorema 3. De forma similar, o estudo de estabilidade com taxa de decaimento e saída limitada é realizado adicionando (27) e (28) aos Teoremas 2 ou 4.

Em alguns problemas práticos o valor do controlador $\mathbf{K}$ deve ser limitado para viabilizar sua implementação. A próxima seção aborda uma técnica para limitar o controlador usando uma constante previamente estabelecida.

\subsection{Condição de estabilidade com otimização do ganho do controlador}

Todos os resultados propostos atuam na estabilidade e desempenho do sistema. Porém pode-se ter a necessidade de implementar $\mathbf{K}$ com pequenos valores absolutos. Nesse caso os resultados obtidos até o momento podem ser insatisfatórios. O próximo resultado apresenta uma solução para esse problema.

Teorema 5 ((Assunção et al., 2007)) Dada uma constante $\mu_{0}$, pode-se especificar um limite para a norma do controlador $\mathbf{K}$ através da otimização de $\beta$, de forma que, $\mathbf{K K}^{\prime}<\beta \mathbf{I} / \mu_{0}^{2}$. O valor ótimo de $\beta$ pode ser obtido pela solução do seguinte problema de otimização:

$$
\begin{gathered}
\min \beta \\
{\left[\begin{array}{cc}
\beta \mathbf{I} & \mathbf{Y} \\
\mathbf{Y}^{\prime} & \mathbf{I}
\end{array}\right]>0,} \\
\mathbf{Q}>\mu_{0} \mathbf{I}, \\
\text { (Conjunto de LMIs), }
\end{gathered}
$$

sendo $\mu_{0}>0, \beta>0, \mathbf{K} \neq \mathbf{0} e$ (Conjunto de LMIs) definido por (5) e (6), ou (10) e (11) para sistemas determinísticos, ou (20) e (21), ou (23) e (24) para sistemas incertos. Podendo ainda incluir as restrições (27) e (28).

Prova: Aplicando o complemento de Schur em (29) tem-se

$$
\mathbf{Y} \mathbf{Y}^{\prime}<\beta \mathbf{I}
$$

Multiplicando (30) à esquerda e à direita por $\sqrt{\mathbf{Q}}$ chega-se em

$$
\sqrt{\mathbf{Q}} \mu_{0} \mathbf{I} \sqrt{\mathbf{Q}}<\sqrt{\mathbf{Q}} \mathbf{Q} \sqrt{\mathbf{Q}} \Rightarrow \mu_{0} \mathbf{Q}<\mathbf{Q Q} .
$$

Agora multiplicando novamente (30) à esquerda por $\mathbf{K}$ e à direita por $\mathbf{K}^{\prime}$ chega-se em

$$
\mathbf{K} \mu_{0} \mathbf{I K}^{\prime}<\mathbf{K Q} \mathbf{K}^{\prime}
$$

Então de (30), (31), (32) e (33) pode-se obter a expressão

$$
\mathbf{K} \mu_{0} \mathbf{I K}^{\prime}<\mathbf{K Q K}^{\prime}<\mathbf{K Q Q K}^{\prime} / \mu_{0}=\mathbf{Y} \mathbf{Y}^{\prime} / \mu_{0}<\beta \mathbf{I} / \mu_{0} .
$$

$\operatorname{Logo} \mathbf{K K}^{\prime}<\beta \mathbf{I} / \mu_{0}^{2}$.

Na próxima seção é ilustrada a eficiência deste procedimento de otimização que pode reduzir as dificuldades práticas presentes na implementação do controlador.

\section{EXEMPLOS}

Alguns exemplos são resolvidos através de simulação usando a metodologia proposta. Os sistemas determinísticos são resolvidos usando o Teorema 2 e posteriormente adicionando o critério de otimização. É adotado o valor $\mu_{0}=1$ para o uso do critério de otimização. O valor da taxa de decaimento $\gamma$ usado em cada exemplo é arbitrário. As LMIs (27) e (28) são adicionadas na resolução de todos os exemplos afim de limitar as saídas dos sistemas. São comparadas as soluções obtidas usando o critério de otimização com as soluções usando apenas o Teorema 2. Para cada exemplo são exibidas as respostas dinâmicas dos sistemas para uma determinada condição inicial. No Exemplo 3 um sistema sujeito a falhas estruturais é modelado como um sistema incerto e resolvido com o Teorema 3, é exibido o comportamento dinâmico do sistema controlado. As simulações dos exemplos são realizadas com o pacote "LMI control toolbox" presente no software MATLAB (Gahinet et al., 1995).

\subsection{Exemplo 1 - Sistema Mecânico}

Como já foi dito a motivação para esse trabalho está na resolução de sistemas mecânicos para controle de vibrações. Um exemplo desses sistemas pode ser visto na Figura 1. 


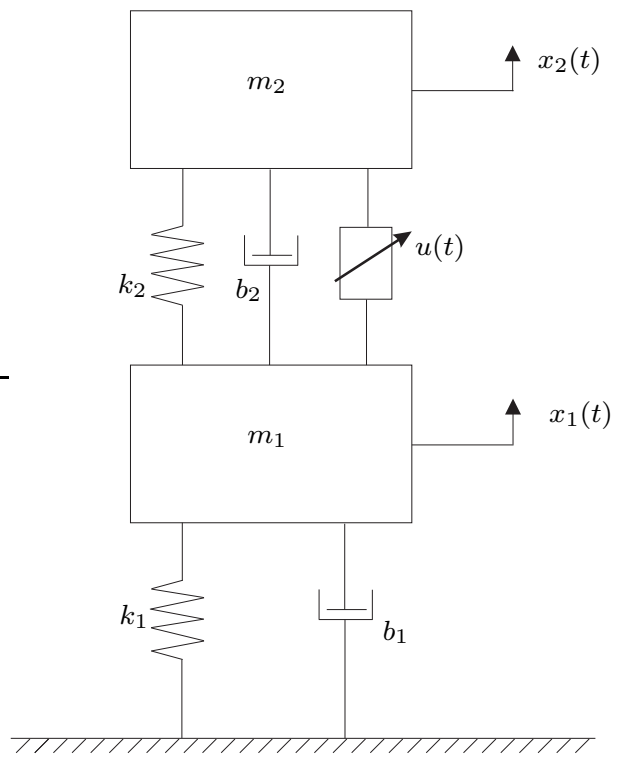

Figura 1: Sistema Mecânico.

O problema é representado matematicamente pelo seguinte sistema de equações diferenciais (Abdelaziz and Valášek, 2004)

$$
\begin{gathered}
{\left[\begin{array}{c}
\dot{x}_{1}(t) \\
\dot{x}_{2}(t) \\
\dot{x}_{3}(t) \\
\dot{x}_{4}(t)
\end{array}\right]=\left[\begin{array}{cccc}
0 & 0 & 1 & 0 \\
0 & 0 & 0 & 1 \\
\frac{-k_{1}-k_{2}}{m_{1}} & \frac{k_{2}}{m_{1}} & \frac{-b_{1}-b_{2}}{m_{1}} & \frac{b_{2}}{m_{1}} \\
\frac{k_{2}}{m_{2}} & \frac{-k_{2}}{m_{2}} & \frac{b_{2}}{m_{2}} & \frac{-b_{2}}{m_{2}}
\end{array}\right]\left[\begin{array}{l}
x_{1}(t) \\
x_{2}(t) \\
x_{3}(t) \\
x_{4}(t)
\end{array}\right]} \\
+ \\
+\left[\begin{array}{c}
0 \\
0 \\
\frac{-1}{m_{1}} \\
\frac{1}{m_{2}}
\end{array}\right] u(t),
\end{gathered}
$$

sendo $k_{1}$ e $k_{2}$ coeficientes de elasticidade das molas, $b_{1}$ e $b_{2}$ são os coeficientes de amortecimento, $x_{1}(t)$ e $x_{2}(t)$ representam o deslocamento vertical dos corpos $m_{1}$ e $m_{2}$ respectivamente. $\mathrm{O}$ vetor de estados é dado por $x(t)=\left[\begin{array}{llll}x_{1}(t) & x_{2}(t) & x_{3}(t) & x_{4}(t)\end{array}\right]^{\prime}$, sendo $x_{3}(t)=$ $\dot{x}_{1}(t)$ e $x_{4}(t)=\dot{x}_{2}(t)$.

Para resolver o problema foram usados os seguintes valores nos parâmetros do problema: $m_{1}=100 \mathrm{~kg}, m_{2}=10 \mathrm{~kg}$, $k_{1}=360 \mathrm{kN} / \mathrm{m}, k_{2}=36 \mathrm{kN} / \mathrm{m}, b_{1}=70 \mathrm{Ns} / \mathrm{m}, b_{2}=$ $50 \mathrm{Ns} / \mathrm{m}$. Neste sistema deseja-se controlar as oscilações em $m_{1}$ usando $m_{2}$ como um atenuador de vibrações.

Supõe-se que a saída de interesse do sistema é dada por:

$$
\left[\begin{array}{l}
y_{1}(t) \\
y_{2}(t)
\end{array}\right]=\left[\begin{array}{llll}
1 & 0 & 0 & 0 \\
0 & 0 & 1 & 0
\end{array}\right]\left[\begin{array}{l}
x_{1}(t) \\
x_{2}(t) \\
x_{3}(t) \\
x_{4}(t)
\end{array}\right]
$$

Adotou-se o valor de $\gamma=3$ para a taxa de decaimento, $\xi_{0}=70$ para o limitante da saída do sistema. Foi usado o valor $\mathbf{x}(0)=\left[\begin{array}{llll}0.15 & 0.15 & 0 & 0\end{array}\right]^{\prime}$ para a condição inicial.

As soluções encontradas pelo Teorema 2 foram

$$
\begin{aligned}
& \mathbf{Q}=\left[\begin{array}{cccc}
1.442 & 2.143 & -18.181 & 4.252 \times 10^{2} \\
2.143 & 85.365 & -1.6 \times 10^{2} & -2.343 \times 10^{3} \\
-18.181 & -1.6 \times 10^{2} & 4.288 \times 10^{3} & -8.51 \times 10^{3} \\
4.252 \times 10^{2} & -2.343 \times 10^{3} & -8.51 \times 10^{3} & 3.899 \times 10^{5}
\end{array}\right] \\
& \mathbf{Y}=\left[\begin{array}{llll}
-1.134 \times 10^{2} & 2.6794 \times 10^{4} & 1.1431 \times 10^{5} & -1.172 \times 10^{6}
\end{array}\right] .
\end{aligned}
$$

O controlador $\mathbf{K}$ obtido foi:

$$
\mathbf{K}=\left[\begin{array}{llll}
-5.283 \times 10^{2} & 4.396 \times 10^{2} & 43.119 & 1.154
\end{array}\right] .
$$

Como já mencionado, o objetivo é encontrar uma solução $\mathbf{K}$ tal que $\operatorname{det}(\mathbf{I}+\mathbf{B K}) \neq 0$. Essa condição é verificada pois, $\operatorname{det}(\mathbf{I}+\mathbf{B K})=0.68416$. A simulação do sistema controlado usando a condição inicial $\mathbf{x}(0)$ pode ser vista na Figura 2. Pela figura pode-se ver que o sistema entrou em regime permanente em menos de $1 s$, satisfazendo a restrição de taxa de decaimento $\gamma=3$, o valor máximo de saída do sistema $\left(\max \sqrt{\mathbf{y}^{\prime}(t) \mathbf{y}(t)}\right)$ é aproximadamente $8.1338<70=\xi_{0}$. Logo todas as restrições de desempenho são atendidas com o controlador projetado. Para reduzir o problema da implementação do controlador, o critério de otimização, (29) e (30), foi adicionado ao Teorema 2. As soluções ótimas obtidas foram

$$
\begin{aligned}
\mathbf{Q} & =\left[\begin{array}{cccc}
1.153 & -0.948 & -3.46 & 58.422 \\
-0.948 & 11.662 & -52.731 & -35.002 \\
-3.46 & -52.731 & 4.8975 \times 10^{3} & -7.643 \times 10^{3} \\
58.422 & -35.002 & -7.643 \times 10^{3} & 4.531 \times 10^{4}
\end{array}\right], \\
\mathbf{Y} & =\left[\begin{array}{llll}
65.509 & 6.464 \times 10^{2} & -1.815 & -0.783
\end{array}\right], \\
\mathbf{K} & =\left[\begin{array}{cccc}
113.14 & 68.972 & 0.9197 & 0.0625
\end{array}\right] \mathrm{e} \\
\beta & =4.2233 \times 10^{5} .
\end{aligned}
$$

Observe que o módulo dos elementos do novo controlador K são menores que o módulo dos elementos do controlador encontrado usando apenas o Teorema 2. A simulação do sistema (3) usando o controlador ótimo com a condição inicial $\mathbf{x}(0)$, pode ser vista na Figura 3. Pela figura podese observar que o sistema continua atendendo a todas as restrições de desempenho.

\subsection{Exemplo 2 - Sistema multivariável}

Considere o sistema (1) multivariável, com matrizes $\mathbf{A}, \mathbf{B}$ e C dadas por:

$$
\mathbf{A}=\left[\begin{array}{lll}
1 & 0 & 0 \\
0 & 3 & 1 \\
2 & 0 & 6
\end{array}\right], \quad \mathbf{B}=\left[\begin{array}{ll}
1 & 0 \\
0 & 1 \\
1 & 1
\end{array}\right], \quad \mathbf{C}=\left[\begin{array}{lll}
1 & 0 & 0 \\
0 & 1 & 0
\end{array}\right]
$$




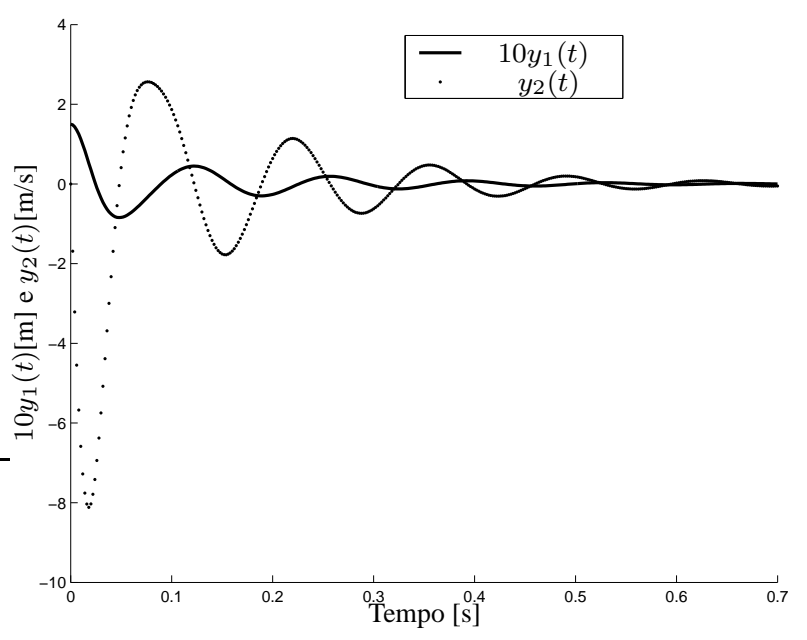

Figura 2: Simulação do sistema com controlador factível.

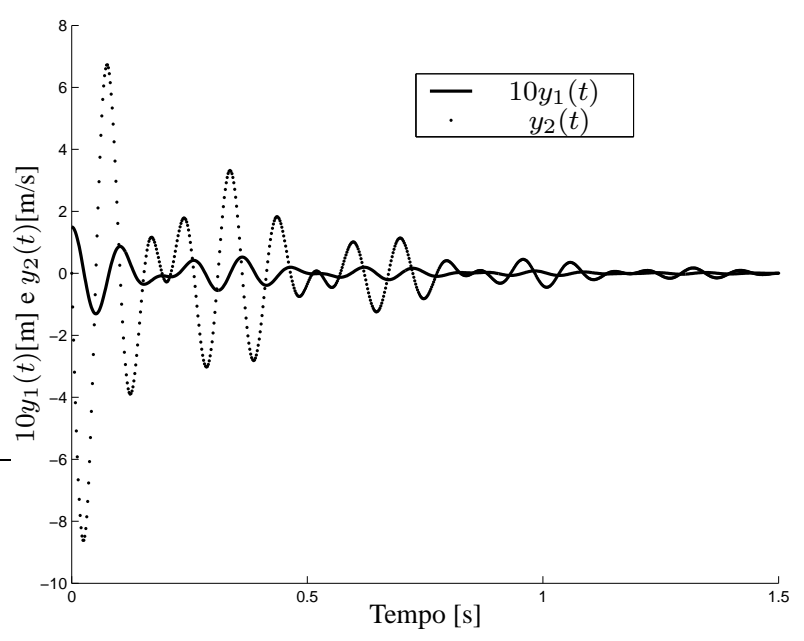

Figura 3: Simulação do sistema com controlador ótimo.

O problema é resolvido usando taxa de decaimento $\gamma=$ 5.5 , controle de saída $\xi_{0}=10$ e condição inicial $\mathbf{x}(0)=$ $\left[\begin{array}{lll}1 & 0 & 0\end{array}\right]^{\prime}$.

Usando o Teorema 2 foram encontrados os seguintes resultados.

$$
\begin{aligned}
& \mathbf{Q}=\left[\begin{array}{ccc}
89.597 & 2.0697 & 64.837 \\
2.0697 & 83.97 & 77.294 \\
64.837 & 77.294 & 122.81
\end{array}\right], \\
& \mathbf{Y}=\left[\begin{array}{ccc}
-95.792 & -1.521 & -66.797 \\
-4.491 & -112.28 & -101.24
\end{array}\right]
\end{aligned}
$$

O controlador $\mathbf{K}$ obtido foi:

$$
\mathbf{K}=\left[\begin{array}{lll}
-1.249 & -0.222 & 0.255 \\
-0.334 & -1.741 & 0.447
\end{array}\right] .
$$

Neste caso $\operatorname{det}(\mathbf{I}+\mathbf{B K}) \simeq-0.006$. Já o controlador ótimo obtido adicionando o critério de otimização foi:

$$
\mathbf{K}=\left[\begin{array}{lll}
-1.249 & -0.272 & 0.274 \\
-0.072 & -1.272 & 0.261
\end{array}\right]
$$

As simulações dos sistemas controlados podem ser vistas nas Figuras 4 e 5. Repare que ambos os sistemas atendem aos índices de desempenho. Tendo alcançado o valor de regime permanente em menos de 1s. O valor máximo de saída é aproximadamente $\sqrt{\mathbf{y}^{\prime}(t) \mathbf{y}(t)}=1.6<10$. O controlador ótimo encontrado possui alguns elementos maiores em módulo que o controlador do Teorema 2. Porém ao avaliar o controlador usando o conceito de norma matricial chega-se nos seguintes resultados:

Norma do controlador usando apenas o $\quad\|\mathbf{K}\|=1.936$, Teorema 2:

Norma do controlador usando o $\|\mathbf{K}\|=1.486$.

Teorema 2 e o critério de otimização:

Verificando que o controlador $\mathbf{K}$ ótimo é capaz de controlar o sistema (3) usando um ganho inferior.

\subsection{Exemplo 3 - Sistema incerto}

Sistemas mecânicos são naturalmente expostos a desgastes físicos e em alguns casos podem ocorrer falhas mecânicas. Considere o sistema mecânico da Figura 1. Suponha que o amortecedor $b_{2}$ sofra uma sobrecarga de operação após $t_{f}$ segundos e quebre. Esse problema pode ser modelado como um sistema incerto (17). Os politopos do sistema são dados por:

- Como já mencionado no Exemplo 4.1, enquanto o sistema funciona normalmente ele pode ser representado pelos parâmetros:

$$
\mathbf{A}_{1}=\left[\begin{array}{cccc}
0 & 0 & 1 & 0 \\
0 & 0 & 0 & 1 \\
\frac{-k_{1}-k_{2}}{m_{1}} & \frac{k_{2}}{m_{1}} & \frac{-b_{1}-b_{2}}{m_{1}} & \frac{b_{2}}{m_{1}} \\
\frac{k_{2}}{m_{2}} & \frac{-k_{2}}{m_{2}} & \frac{b_{2}}{m_{2}} & \frac{-b_{2}}{m_{2}}
\end{array}\right], \mathbf{B}=\left[\begin{array}{c}
0 \\
0 \\
\frac{-1}{m_{1}} \\
\frac{1}{m_{2}}
\end{array}\right] .
$$

- Após $t_{f}$ segundos o amortecedor $b_{2}$ quebra e o sistema dinâmico passa a ser representado por:

$$
\mathbf{A}_{2}=\left[\begin{array}{cccc}
0 & 0 & 1 & 0 \\
0 & 0 & 0 & 1 \\
\frac{-k_{1}-k_{2}}{m_{1}} & \frac{k_{2}}{m_{1}} & \frac{-b_{1}}{m_{1}} & 0 \\
\frac{k_{2}}{m_{2}} & \frac{-k_{2}}{m_{2}} & 0 & 0
\end{array}\right], \quad \mathbf{B}=\left[\begin{array}{c}
0 \\
0 \\
\frac{-1}{m_{1}} \\
\frac{1}{m_{2}}
\end{array}\right] .
$$

O problema é resolvido com os mesmos valores do Exemplo 4.1. Usando o MATLAB para resolver as LMIs (20) e (21) 


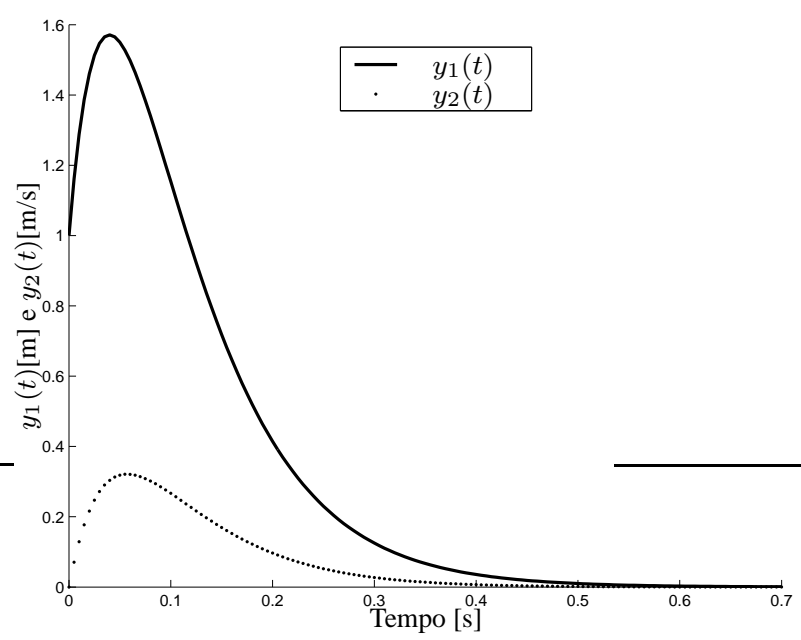

Figura 4: Simulação do sistema com controlador factível.

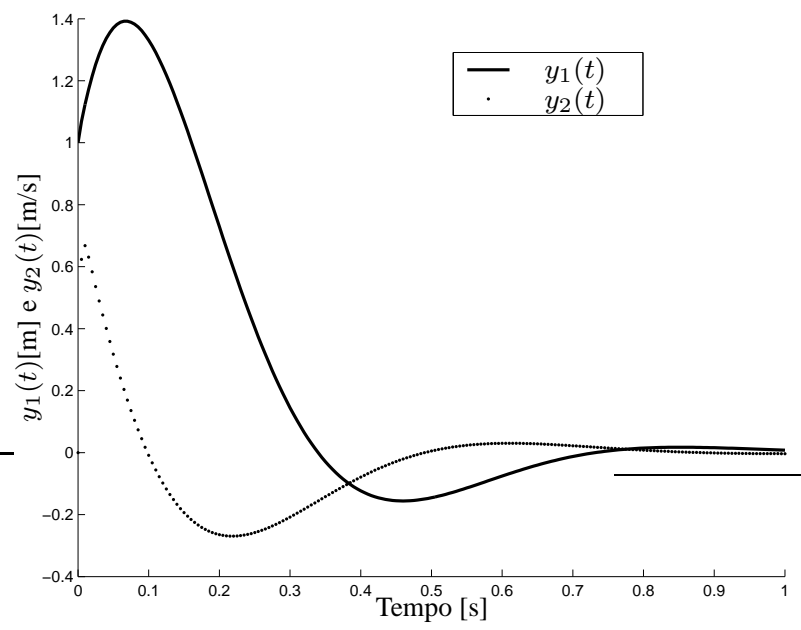

Figura 5: Simulação do sistema com controlador ótimo.

do Teorema 3, a seguinte solução foi encontrada:

$$
\begin{gathered}
\mathbf{Q}=\left[\begin{array}{cccc}
23.188 & 21.747 & -37.149 & 363.15 \\
21.747 & 27.188 & -346.39 & -532.81 \\
-37.149 & -346.39 & 7.7476 \times 10^{4} & 5.8687 \times 10^{4} \\
363.15 & -532.81 & 5.8687 \times 10^{4} & 1.935 \times 10^{5}
\end{array}\right], \\
\mathbf{Y}=\left[\begin{array}{llll}
405.37 & 9.4244 \times 10^{3} & -6.3438 \times 10^{5} & -1.7602 \times 10^{6}
\end{array}\right] .
\end{gathered}
$$

De (22) um controlador que resolve o problema é

$$
\mathbf{K}=\left[\begin{array}{llll}
-134.45 & 288.81 & -1.1218 & -7.709
\end{array}\right] \text {. }
$$

sendo $\operatorname{det}(\mathbf{I}+\mathbf{B K})=0.24032 \neq 0$.

Pela Figura 6 o sistema mecânico (35) representado pelas matrizes $A_{1}$ e $B$ é naturalmente estável. Agora observe o comportamento dinâmico do sistema (35) quando o amortecedor $b_{2}$ quebra no instante $t_{f}=2 \mathrm{~s}$, após essa falha o sistema passa a ser representado pelas matrizes

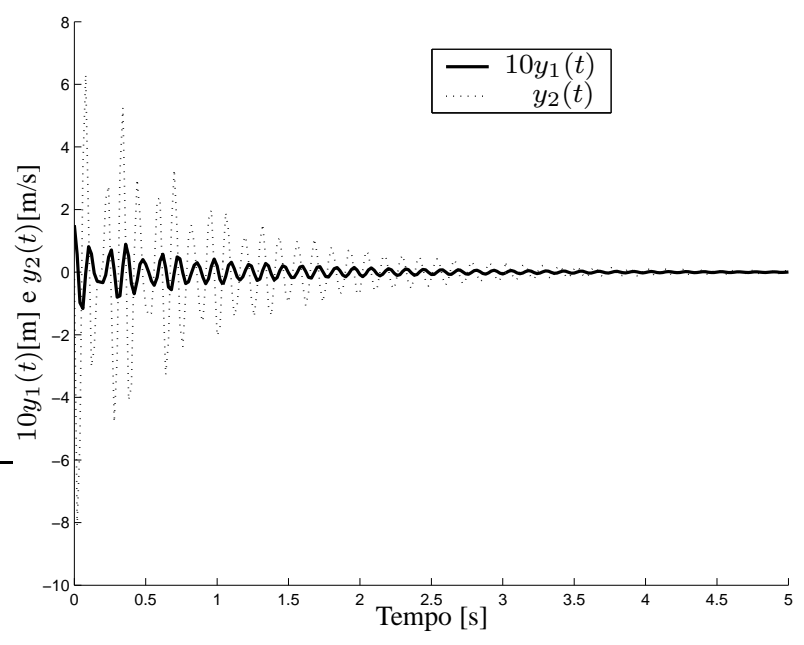

Figura 6: Simulação do sistema (35).

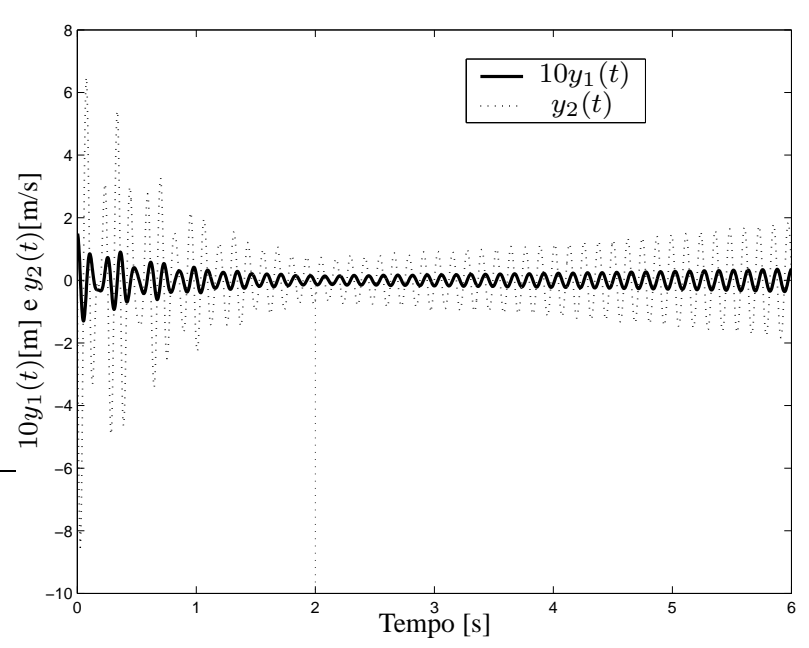

Figura 7: Simulação do sistema (35) com falha do amortecedor em $2 \mathrm{~s}$.

$A_{2}$ e $B$, e com isso o sistema se torna instável (Figura 7). Esse problema é resolvido ao realimentar o sistema com o controlador (36). O comportamento dinâmico do sistema controlado é visto na Figura 8, repare que o sistema permanece estável mesmo após a quebra do amortecedor em $t_{f}=2 \mathrm{~s}$.

\section{CONCLUSÕES}

A técnica proposta representa uma nova ferramenta para o uso da realimentação da derivada dos estados em sistemas lineares determinísticos ou incertos. É mais versátil que outras técnicas encontradas na literatura pois é capaz de resolver problemas com índices de desempenho, inclusive sistemas sujeitos à falhas estruturais, sem o uso de técnicas 


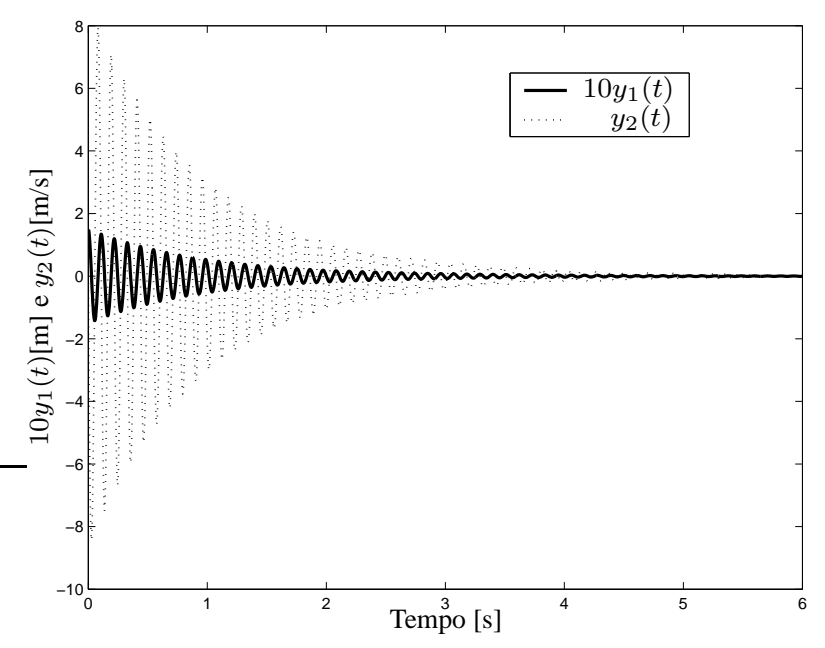

Figura 8: Simulação do sistema dinâmico controlado.

adicionais de controle. Bastando fornecer os parâmetros de taxa de decaimento $\gamma$ e limitante do valor de saída $\xi_{0}$. A modelagem do problema em LMIs tornou mais fácil o tratamento de estabilidade assintótica do sistema, pois LMIs quando factíveis, são facilmente resolvidas em microcomputadores. O critério de otimização apresentado é simples de ser incorporado em projetos envolvendo LMIs e se mostrou eficiente nos resultados dos exemplos. Outros resultados obtidos posteriormente pelos autores, relativos a esse tema, podem ser encontrados em (Assunção et al., 2007; Cardim et al., 2007).

\section{AGRADECIMENTOS}

Agradecemos à CAPES, ao CNPQ e à FAPESP pelo apoio financeiro.

\section{REFERÊNCIAS}

A. Bunse-Gerstner, R. Byers, V. M. and Nichols, N. (1999). Feedback design for regularizing descriptor systems , Linear Algebra and its Applications 299(1-3): 119151.

Abdelaziz, T. H. S. and Valášek, M. (2004). Pole-placement for siso linear systems by state-derivative feedback, IEE Proceedings-Control Theory and Applications 151(4): 377-385.

Assunção, E., Teixeira, M. C. M., Faria, F. A., da Silva, N. A. P. and Cardim, R. (2007). Robust state-derivative feedback LMI-based designs for multivariable linear systems, International Journal of Control 80(8): 12601270.
Assunção, E., Andrea, C. Q. and Teixeira, M. C. M. (2007). $\mathcal{H}_{2}$ and $\mathcal{H}_{\infty}$-optimal control for the tracking problem with zero variation, IET Control Theory Applications 1(3): 682-688.

Assunção, E., Marchesi, H. F., Teixeira, M. C. M. and Peres, P. L. D. (2007). Global Optimization for the $\mathcal{H}_{\infty}$-Norm Model Reduction Problem, International Journal of Systems Science 38(2): 125-138.

Assunção, E. and Teixeira, M. (2001). Projeto de sistemas de controle via LMIS usando o MATLAB, in J. Balthazar, V. Oliveira, G. Silva and J. Rosário (eds), Aplicações em Dinâmica e Controle, São Carlos, pp. 350-368.

Boyd, S., El Ghaoui, L., Feron, E. and Balakrishnan, V. (1994). Linear matrix inequalities in systems and control theory, Vol. 15 of SIAM studies in applied mathematics, 2nd edn, SIAM Studies in Applied Mathematics, Philadelphia.

Bunse-Gerstner, A., Mehrmann, V. and Nichols, N. (1992). Regularization of descriptor systems by derivative and proportional state feedback, SIAM journal on matrix analysis and applications 13(1): 46-67.

Cardim, R., Teixeira, M. C. M., Assunção, E. and Covacic, M. R. (2007). Design of state-derivative feedback controllers using a state feedback control design, 3rd IFAC Symposium on System, Structure and Control, Vol. 1, Iguassu Falls, PR, Brazil, pp. 6, Article 135.

Cardim, R., Teixeira, M. C. M., Assunção, E. and Faria, F. A. (2008). Systems, Structure and Control, In-Teh, Vienna, Austria, chapter Control Designs for Linear Systems Using State-Derivative Feedback, pp. 1-28.

URL: $h t t p: / / w w w . s t a n f o r d . e d u / \sim$ boyd/lmibook/lmibook.pdf

Chen, C. (1999). Linear system theory and design, The Oxford series in electrical and computer engineering, 3rd edn, Oxford, New York.

de Oliveira, M. C., Farias, D. P. and Geromel, J. C. (1997). LMISol, User's guide, UNICAMP, Campinas-SP, Brasil. http://www.dt.fee.unicamp.br/mauricio/software.html.

Dorf, R. C. and Bishop, R. H. (2001). Sistemas de controle modernos, 8 edn, LTC(Livros Técnivos e Científicos Editora S.A.), Rio de Janeiro.

Duan, G. R., Irwin, G. W. and Liu, G. P. (1999). Robust stabilization of descriptor linear systems via proportional-plus-derivative state feedback, Proceedings of the 1999 American Control Conference, American Control Conference, American Automatic Control Council, San Diego, pp. 1304-1308. 
E. Reithmeier and G. Leitmann (2003). Robust Vibration Control of Dynamical Systems Based on the Derivative of the State, Archive of Applied Mechanics 72(1112): $856-864$.

Faria, F. A., Assunção, E., Teixeira, M. C. M., Cardim, R. and da Silva, N. A. P. (2009). Robust state-derivative pole placement LMI-based designs for linear systems, International Journal of Control 82(1): 1-12.

Gahinet, P., Nemirovski, A., Laub, A. J. and Chilali, M. (1995). LMI control toolbox - For use with Matlab, The Math Works Inc. http://www.mathworks.com/.

Garcia-Planas, M. (2003). Regularizing generalized linear systems by means a derivative feedback, Physcon 2003 Proceedings, Physics and Control, IEEE. Proceedings, St. Petersburg, pp. 1134-1140.

Isermann, R. (1997). Supervision, fault-detection and fault-diagnosis methods - an introduction, Control Engineering Practice 5(5): 639-652.

Isermann, R. and Ballé, P. (1997). Trends in the application of model-based fault detection and diagnosis of technical processes, Control Engineering Practice 5(5): 709-719.

Lewis, F. and Syrmos, V. (1991). A geometric theory for derivative feedback, IEE Trans. Autom. Control 36(9): 1111-1116.

Meyer, C. D. (2000). Matrix analysis and applied linear algebra, SIAM Studies in Applied Mathematics, Philadelphia. http://www.matrixanalysis.com/DownloadChapters.html.

Ogata, K. (2000). Engenharia de controle moderno, 3 edn, LTC(Livros Técnivos e Científicos Editora S.A.), Rio de Janeiro.

S. K. Kwak and G. Washington and R. K. Yedavalli (2002a). Acceleration-Based Vibration Control of Distributed Parameter Systems Using the "Reciprocal StateSpace Framework", Journal of Sound and Vibration 251(3): 543-557.

S. K. Kwak and G. Washington and R. K. Yedavalli (2002b). Acceleration Feedback-Based Active and Passive Vibration Control of Landing Gear Components, Journal of Aerospace Engineering 15(1): 1-9.

Teixeira, M. C. M., Assunção, E. and Avellar, R. G. (2003). On Relaxed LMI-Based Designs for Fuzzy Regulators and Fuzzy Observers, IEEE Transactions on Fuzzy Systems 11(5): 613-623.
Teixeira, M. C. M., Assunção, E., Cardim, R. and Covacic, M. R. (2006). Realimentação da Derivada de Estados a Partir do Projeto com Realimentação de Estados, Anais do XVI Congresso Brasileiro de Automática (CBA), Vol. 1, XVI Congresso Brasileiro de Automática, Salvador, BA, Brasil, pp. 726-731.

Teixeira, M. C. M., Assunção, E. and Palhares, R. M. (2005). Discussion on: $\mathcal{H}_{\infty}$ Output Feedback Control Design for Uncertain Fuzzy Systems with Multiple Time Scales: An LMI Approach, European Journal of Control 11(2): 167-169.

Teixeira, M. C. M., Covacic, M. R. and Assunção, E. (2006). Design of SPR Systems with Dynamic Compensators and Output Variable Structure Control, International Workshop on Variable Structure Systems, Vol. 1, Proceeding of the 2006 International Workshop on Variable Structure Systems, Alghero-Itália, pp. 328333.

Y. F. Duan and Y. Q. Ni and J. M. Ko (2005). State-Derivative feedback Control of Cable Vibration Using Semiactive Magnetorheological Dampers, Computer-Aided Civil and Infrastructure Engineering 20(6): 431-449. 